\title{
INVESTIGACIÓN
}

\author{
Recibido: 13/05/2019 --- Aceptado: 24/06/2019 --- Publicado: 15/03/2020
}

\section{RETOS ANTE LA REESCRITURA DEL CUERPO SEXUADO DE LA MUJER Y LA PROSTITUCIÓN FEMENINA: ESTUDIO DE CASO}

\author{
Challenges before rewriting the women's sexed body and female prostitution: \\ a case study
}

D 81 $\mathbb{R}^{6}$ Antonio Jesús Martínez Pleguezuelos. Universidad Complutense de Madrid. España.

antmar17@ucm.es

(iD) 88 $\mathbb{R}^{\mathrm{C}}$ Elena Alcalde Peñalver. Universidad de Alcalá. España. e.alcalde@uah.es

D (8) Alexandra Santamaría Urbieta. Universidad de Alcalá. España.

alexandra.santamaria@uah.es

\section{RESUMEN}

Este estudio pretende poner de manifiesto los desafíos que se afrontan en la práctica profesional de la traducción a la hora de reconocer la perspectiva feminista como un ámbito en el que necesitamos poner el foco de atención cuando se trabaja con textos relacionados con la prostitución. Sabedores de las dificultades que entraña una traducción que sitúe en pie de igualdad a hombre y mujer en el tratamiento de la prostitución, planteamos a un grupo de estudiantes sin formación específica previa en traducción un ejercicio práctico en el que debían traducir de inglés a español un artículo que aborda las complejidades sociales y culturales que emanan de concebir la prostitución en tanto que derecho humano. El objetivo de la actividad consistía en favorecer una reflexión sobre las implicaciones ideológicas que participan en la traducción de este tipo de textos, así como conocer el punto de vista y las herramientas de las que disponían para un encargo de estas características. El estudio se llevó a cabo en tres fases. Tras el análisis cualitativo de los resultados obtenidos corroboramos las implicaciones éticas del ejercicio de la traducción en la definición discursiva tanto de la mujer como del hombre en sentidos opuestos. Por ello, consideramos necesario seguir formando a estudiantes en traducción para que sean capaces de detectar las herramientas discursivas que contribuyen a afianzar los desequilibrios entre hombres y mujeres y los roles que quedan asignados en la sociedad.

\footnotetext{
${ }^{1}$ Antonio Martínez Pleguezuelos: Doctor en Traducción por la Universidad de Salamanca, Profesor e Investigador de la Universidad Complutense de Madrid.
} 
Martínez Pleguezuelos, A. J.; Alcalde Peñalver, E. y Santamaría Urbieta, A.

Retos ante la reescritura del cuerpo sexuado de la mujer y la prostitución femenina: estudio de caso

PALABRAS CLAVE: traducción - perspectiva femenina - prostitución - discurso ideología - texto - reescritura.

\begin{abstract}
This study brings to light the challenges that translators have to face when identifying the need of bringing the feminist perspective into focus if faced with texts related with the topic of prostitution. The case study here described highlights a practical exercise given to a group of students in which they had to translate from English into Spanish an article that tackled the social and cultural complexities that come from conceiving prostitution as a human right. The goal of this activity consisted, on the one hand, in favouring a reflection about the ideological implications that participate in the translation of this type of texts and, on the other hand, in getting to know the point of view as well as the tools the students had when facing a translation like this. After the analysis of the results obtained, we verify the ethical implications of the translation activity in the discursive definition of both the woman and man in opposed senses. Therefore, we consider it necessary to continue training students in translation so that they are able to detect the discursive tools that contribute to consolidating the imbalances between men and women and the roles that they are assigned in society.
\end{abstract}

KEY WORDS: translation - feminist perspective - prostitution - discourse ideology - text - rewriting.

\title{
RETOS DIANTE DA REESCRITURA DO CORPO SEXUADO DA MULHER E A PROSTITUIÇAO FEMININA: ESTUDO DO CASO
}

\section{RESUME}

Este estudo pretende pôr em manifesto os desafios que afrontam na pratica profissional da tradução na hora de reconhecer a perspectiva feminista como um âmbito no qual necessitamos pôr o foco de atenção quando se trabalha com textos relacionados à a prostituição. Sabedores das dificuldades que entranha uma tradução que situe em igualdade a homens e mulheres no tratamento da prostituição, propusemos a um grupo de estudantes sem formação específica prévia em tradução um exercício prático no qual deviam traduzir do inglês ao espanhol um artigo que aborda as complexidades sociais e culturais que emanam de conceber a prostituição como um direito humano. O objetivo da atividade consistia em favorecer uma reflexão sobre as implicações ideológicas que participam na tradução deste tipo de textos, assim como conhecer o ponto de vista e as ferramentas das que dispunham para uma incumbência destas características. O estudo foi feito em 3 fases. Depois da analises qualitativa dos resultados obtidos corroboramos as implicações éticas do exercício da tradução na definição discursiva tanto da mulher como do homem em sentidos opostos. Por isso, consideramos necessário seguir formando a estudantes em tradução para que sejam capazes de detectar as ferramentas discursivas que 
Martínez Pleguezuelos, A. J.; Alcalde Peñalver, E. y Santamaría Urbieta, A.

Retos ante la reescritura del cuerpo sexuado de la mujer y la prostitución femenina: estudio de caso

contribuem a assegurar os desequilíbrios entre homens e mulheres e os papéis que ficam assignados na sociedade.

PALAVRAS CHAVE: tradução - perspectiva feminina - prostituição - discurso ideologia - texto - reescrita.

\section{Como citar el artículo:}

Martínez Pleguezuelos, A. J.; Alcalde Peñalver, E. y Santamaría Urbieta, A. (2020). Retos ante la reescritura del cuerpo sexuado de la mujer y la prostitución femenina: estudio de caso. [Challenges before rewriting the women's sexed body and female prostitution: a case study].Vivat Academia. Revista de Comunicación, 150, 59-80. doi: http:/ / doi.org/10.15178/va.2020.150.59-80

Recuperado de http://www.vivatacademia.net/index.php/vivat/article/view/1184

\section{INTRODUCCIÓN}

Nos encontramos en un momento histórico en el que la mujer alza la voz para reivindicar sus derechos y denunciar una discriminación transversal: techo de cristal, salarios inferiores a los de los hombres, abusos de todo tipo, estereotipos, prejuicios, así como jóvenes convertidas en objeto de consumo, entre otros. Instituciones a nivel nacional e internacional hacen pública una realidad que debe hacerse visible también en las universidades, por lo que como docentes debemos conocer la realidad de nuestras aulas y preparar a los futuros profesionales de los distintos ámbitos laborales. En el caso concreto que nos ocupa en las siguientes páginas, nos aproximamos a la figura del traductor y analizamos la reescritura de textos actuales con marcadas tendencias ideológicas o simbolismos nacidos en el seno de la sociedad en la que vivimos.

El estudio de caso que planteamos a continuación describe un ejercicio práctico que se presentó a un grupo de estudiantes del primer curso del Grado en Lenguas Modernas y Traducción de la Universidad de Alcalá. Se trata de un encargo en el que debían traducir de inglés a español un artículo periodístico de la versión online de The Guardian que aborda las complejidades sociales y culturales que emanan de concebir la prostitución en tanto que derecho humano. Para ello, y con el objetivo de favorecer una reflexión sobre las implicaciones ideológicas que participan en la traducción de este tipo de textos, así como conocer su punto de vista y las herramientas de las que disponían y con las que partían para un encargo de estas características, este proceso se dividió en tres pasos: (1) pre-traducción, (2) traducción propiamente dicha y (3) post-traducción. Esta división está relacionada con las metas que se quieren alcanzar, detalladas más adelante, vinculadas todas ellas a conseguir un replanteamiento por parte de los estudiantes de la posición central del traductor a la hora de reescribir realidades sociales que afectan a la imagen de un determinado colectivo, en este caso el de aquellas mujeres que se dedican a la prostitución. 
Martínez Pleguezuelos, A. J.; Alcalde Peñalver, E. y Santamaría Urbieta, A.

Retos ante la reescritura del cuerpo sexuado de la mujer y la prostitución femenina: estudio de caso

El reto que supone enfrentarse a una traducción de tales características proviene de la toma de perspectiva que se adopta al aproximarse a las dificultades intrínsecas que contiene. Las repercusiones que se derivan de reescribir un discurso sobre el tratamiento de la prostitución, la figura de la mujer y la manipulación de su cuerpo, así como la participación velada del hombre en el mercado sexual son evidentes a la vista de los trabajos desarrollados en el seno del movimiento feminista. Este estudio pretende poner de manifiesto los desafíos de la práctica profesional de la traducción desde una perspectiva didáctica. Tanto estudiantes como docentes deben reconocer la perspectiva feminista como un ámbito en el que necesitamos poner el foco de atención a la hora de reescribir la figura del cuerpo de la mujer, así como de los textos relacionados con la prostitución femenina.

\subsection{La entidad del cuerpo sexuado de la mujer}

Ante las elevadas cotas de visibilidad que ha alcanzado el mercado de la prostitución en gran parte del mundo y el creciente debate social generado en torno a esta actividad, las visiones polarizadas que defienden la legalización de la prostitución o su erradicación se encuentran lejos de alcanzar un acuerdo satisfactorio para ambas posturas. Hoy en día, el enfrentamiento entre las dos posiciones ha trascendido el plano de la teoría feminista y al ámbito académico se han sumado opiniones de la propia industria del sexo, la filosofía, los medios de comunicación o la política a escala nacional e internacional. A tal efecto, parece oportuno señalar que el informe emanado de la Dirección General de Políticas Internas del Parlamento Europeo "Sexual exploitation and prostitution and its impact on gender equality" (Schulze, 2014) establece que, en consonancia con la posición que apoya la erradicación de la explotación sexual, la prostitución no puede considerarse un negocio legítimo por defender un enfoque opuesto a los principios establecidos en la Carta de los Derechos Fundamentales de la Unión Europea, entre los que se encuentra la igualdad de géneros.

En una explicación más pormenorizada, este estudio mantiene que tanto la prostitución como la explotación sexual "are highly gendered issues with in most cases women and girls selling their body, either by coercion or consent, to men or boys who pay for this service" (2014, p. 9). A este respecto, muchos otros trabajos desarrollados sobre el tema reconocen que el debate se genera en una sociedad con grandes asimetrías de poder entre hombres y mujeres. De esta situación de desigualdad, continúa el informe (2014, p. 19), además del refuerzo de los estereotipos de género, se desprende el derecho del hombre a acceder al cuerpo de la mujer y a su sexualidad por su posición superior, y a equiparar las necesidades sexuales del hombre con una especie de poder otorgado únicamente por su género. Así lo expone Gimeno (2014) cuando defiende que "los hombres no compran un cuerpo, ni sexo, sino una fantasía de dominio y masculinidad tradicional", por lo que el contexto de la prostitución puede entenderse, en consonancia con la perspectiva de Eksberg (2004, p. 1190), como un espacio permisivo con el abuso y la violencia hacia la mujer que contribuye a apuntalar una jerarquía dominante masculina frente a una subordinada femenina (cf. Farley, 2004; Mackinnon, 2005). No en vano, el Manifiesto 
Martínez Pleguezuelos, A. J.; Alcalde Peñalver, E. y Santamaría Urbieta, A.

Retos ante la reescritura del cuerpo sexuado de la mujer y la prostitución femenina: estudio de caso

político por la abolición de la prostitución, elaborado por distintas asociaciones en España, defiende que la prostitución, en cualquier circunstancia, es una "modalidad de explotación sexual de las personas prostituidas, [...] y es una de las formas más arraigadas en las que se manifiesta, ejerce y perpetúa la violencia de género" (en Villa Camarma, 2010, p. 170), lo que constituye una más de las múltiples formas de agresión contra la mujer.

Se asimila, de este modo, el concepto de "mujer" en tanto que "cuerpos y trozos de cuerpos de los que es normal disponer y de los que no importa preguntarse cómo ni por qué están ahí" (de Miguel Álvarez, 2012, p. 64), como vía para la legitimación de las relaciones de dominación del hombre sobre el cuerpo desposeído de la mujer. Esta paradoja positivista coincide con la descripción de la construcción material de los cuerpos que lleva a cabo Judith Butler al afirmar que no existe una esencia corporal que escape a las complejas relaciones de poder que se imponen actualmente en las sociedades contemporáneas, y a la que se pueda acceder sin pasar previamente por el tamiz del lenguaje. Tal y como mantiene la filósofa (Butler, 1999/2007, p. 254), el cuerpo se muestra como una superficie pasiva que queda inscrita por redes de poder externas a él, que lo penetran, pero que, al mismo tiempo, desde estos postulados constructivistas, queda en entredicho como entidad prediscursiva. De este modo, el género se convierte en el enclave para reinterpretar determinados significados culturales desde los patrones sociales heredados en torno al género (de Mauro Rucovsky, 2015, p. 44). Como veremos a continuación con los ejemplos seleccionados de las traducciones presentadas por los estudiantes, el tratamiento del léxico será decisivo a la hora de perfilar la identidad de la mujer y su participación en el ejercicio de la prostitución.

No por casualidad, Butler se cuestiona acertadamente si el cuerpo se entiende así como base sobre la que tanto el género como los sistemas de sexualidad obligatoria operan. Mediante el proceso genealógico que retoma de Foucault, la autora insta a descubrir la historia grabada en los cuerpos como prácticas significantes que los someten y que les aportan sentido (Butler, 1999/2007, p. 256 y ss.). Con ello, los valores culturales se inscriben en el cuerpo como una plantilla reguladora que permite comprenderlos, pero al mismo tiempo establece aquellas posibilidades de inteligibilidad que condicionan lo que constituye al cuerpo y cuáles son sus límites (1999/2007, p. 257).

Encontramos, de este modo, cuerpos habitados por una infinidad de discursos que colaboran en su propia definición y que, en buena medida, como plantean los enfoques feministas, han desequilibrado las posiciones entre hombre y mujer al basarse en esquemas que anteponen el derecho del hombre al de la mujer. Por lo tanto, el cuerpo, concebido como última frontera de la subjetividad, cede ante las presiones sociales y culturales y queda abierto a una lectura que configura el perfil de la mujer y, específicamente en nuestro estudio, el de aquellas dedicadas a la prostitución. 
Martínez Pleguezuelos, A. J.; Alcalde Peñalver, E. y Santamaría Urbieta, A.

Retos ante la reescritura del cuerpo sexuado de la mujer y la prostitución femenina:

estudio de caso

\subsection{Reescribir el cuerpo}

Desde estas bases epistemológicas, es fácil pensar en la relevancia de la práctica de la traducción en la construcción del discurso sobre el mercado del sexo. Como hemos visto, y al amparo de las teorías postestructuralistas del lenguaje, habitamos en mundos discursivos y, por tanto, nuestra propia existencia se produce a través de la lengua. Los actos de habla que presentó Austin, base desde la que Butler desarrolla ampliamente su teoría de la performatividad, desplazaron la posición privilegiada de la Verdad hacia el terreno del lenguaje (Burgos Díaz, 2006, p. 99) para descubrir la enorme capacidad de las palabras a la hora de construir realidades.

En un mundo fundamentalmente traducido como el que habitamos, con un contacto incesante e inmediato entre diferentes comunidades sociales, culturales y lingüísticas, el argumento de la traducibilidad de los cuerpos se ha convertido en una de las líneas de investigación de mayor interés en los últimos años (cf. Vidal Claramonte, 2018; Arcos Rodríguez, 2018) por su capacidad para definirlos y moldearlos. En la línea de aquellos autores que se han aproximado al estudio de la identidad a través del texto reescrito (cf. House, Martín Ruano y Baumgarten, 2005; Cronin, 2006; Vidal Claramonte, 2007), los enfoques traductológicos que abordan la definición del cuerpo pretenden mostrar la fuerza performativa del texto reescrito en la representación de cuerpos sexuados.

Este terreno relativamente reciente en el ámbito de los Estudios de Traducción puede explorarse desde un enfoque ampliado en relación con lo que, de forma tradicional, se ha considerado el objeto de trabajo del traductor. Desde la aparición del Giro cultural que presentaron Bassnett y Lefevere (1990) en el que la perspectiva intercultural pasó a ser dominante en el desarrollo del ejercicio de la traducción hasta el Giro del poder descrito por Gentzler (2002, 2012) y Tymozcko (2007), las diversas posiciones que se han mantenido sobre qué es traducir y cuál es el papel del traductor han evolucionado a marchas forzadas en sintonía con los avances y nuevas perspectivas que se incorporan desde otras disciplinas. La gran capacidad del lenguaje para recrear cuerpos e identidades ha hecho replantear los límites de la traducción hasta incluir posicionamientos como, por ejemplo, los que abarca la definición de los Post-Translation Studies que propone Gentzler (2017).

Basta, como muestra, tener presente las llamadas de atención de distintas autoras que desde los Estudios de género y las últimas corrientes feministas avisan del carácter connotado de la figura de la mujer prostituta y del estigma que soportan en el ejercicio de esta actividad, y de cómo, en la gran mayoría de los discursos sobre este debate, la figura y posición del hombre quedan invisibilizadas $u$ obviadas cuando se pretende abordar el problema. Villa Camarma (2010, p. 165) afirma que "hablar de trabajadora sexual implica hablar de clientes. Hablar de prostitutas implica hablar de prostituidores, los intermediarios (proxenetas, chulos y la industria del sexo)". En este sentido, traducir dicha perspectiva supondrá adoptar una posición ideológica determinada que vendrá a afianzar los marcados desequilibrios sociales que siguen cargando contra la mujer y refuerzan la superioridad masculina. 
Martínez Pleguezuelos, A. J.; Alcalde Peñalver, E. y Santamaría Urbieta, A.

Retos ante la reescritura del cuerpo sexuado de la mujer y la prostitución femenina: estudio de caso

En palabras de Gimeno (2014): "la prostitución de hoy adiestra, enseña, disciplina el cuerpo masculino en la desigualdad extrema, en la mercantilización desnuda de las relaciones humanas y erotiza esa relación", y una reescritura que contribuya a perpetuar estereotipos se convertirá en el cómplice perfecto capaz de reproducir dichas desigualdades de género en una lengua diferente para otro público. En el análisis de las distintas traducciones recibidas de los alumnos este punto será fundamental para entender cómo se diseña discursivamente el papel del hombre y de la mujer en el terreno de la prostitución, ya que una reescritura que enfatice la figura de la mujer prostituta frente al ocultamiento del hombre como cliente participará de manera directa en esta jerarquía de poder.

Por el contrario, en consonancia con las perspectivas de género que ya se encuentran afianzadas en el ámbito de la traducción, una reescritura ética que visibilice el papel protagonista del hombre en la prostitución y que arroje luz sobre su participación en esta actividad económica contribuirá a desarrollar un discurso a favor de la igualdad entre géneros sin que se cargue o se estigmatice a la mujer y su sexualidad. La selección léxica que realice el traductor, el énfasis en presentar como sujeto principal a la mujer en la prostitución, la connotación de los verbos escogidos o la posición temática de la información pueden contribuir, de manera irremediable, a presentar la información al público meta de una forma sesgada que afiance las desigualdades tradicionales entre ambos géneros cuando se reescribe el vínculo entre prostituta y cliente.

\section{OBJETIVOS}

Sabedores de las dificultades que entraña una traducción que sitúe en pie de igualdad a hombre y mujer en el tratamiento de la prostitución, hemos planteado una prueba de traducción a estudiantes del primer curso del Grado en Lenguas Modernas y Traducción de la Universidad de Alcalá. Es necesario señalar que los alumnos y alumnas de este primer curso no han recibido aún formación específica en traducción, puesto que las asignaturas iniciales se basan en lengua y cultura como fase previa a una introducción a la traducción que reciben ya en el segundo año de estudios. No obstante, como parte de la asignatura Inglés II en la que los alumnos mostraban constante interés por la profesión del traductor, decidimos diseñar una actividad que, además de implicar el trabajo de comprensión del texto en inglés y su traducción al español, abarcara un ámbito temático que hiciera a los estudiantes detenerse a reflexionar. La actividad se calificó como parte del 20\% de evaluación destinado a participación y a la entrega de diarios de aprendizaje en los que los estudiantes explicaban lo que hacían para el desarrollo de la competencia lingüística en inglés fuera del aula. Los objetivos de nuestro estudio fueron los siguientes:

1. Conocer cómo estudiantes de primero sin formación específica en traducción se enfrentan a problemas de traducción asociados a una temática de género.

2. Analizar la selección léxica de los estudiantes y las diferencias que puedan existir entre la posición temática de las traducciones finales en función de esta selección. 
Martínez Pleguezuelos, A. J.; Alcalde Peñalver, E. y Santamaría Urbieta, A.

Retos ante la reescritura del cuerpo sexuado de la mujer y la prostitución femenina: estudio de caso

3. Generar concienciación entre estudiantes que aún no han recibido formación específica en traducción acerca del papel del traductor en discursos relacionados con perspectivas de género.

\section{METODOLOGÍA}

Para la realización del estudio dividimos nuestra investigación en tres fases. En primer lugar, explicamos al estudiantado en qué consistía la actividad y les pedimos que organizaran el trabajo en parejas. Se formaron por lo tanto 12 grupos al haber un total de 24 participantes en el aula. Antes de proceder a la traducción del artículo, tuvieron que completar un cuestionario con preguntas abiertas que invitaba a una reflexión previa sobre el tema y las posibles dificultades de traducción (véase Anexo 1). Con estas preguntas, debían pensar en los términos, expresiones o partes del texto que, según su opinión, podrían resultar difíciles de traducir sin consultar ningún tipo de recurso lingüístico. Asimismo, una de las cuestiones les pedía que buscaran textos paralelos y que, con su ayuda, aportaran la traducción de algunas palabras específicas del texto. Seguidamente, debían buscar diferentes opciones de traducción de términos del texto en inglés y decantarse por alguna de ellas. Por último, en función de los enfoques teóricos que analizan el componente ideológico en las reescrituras (cf. Calzada, 2003), en el cuestionario se requería que reflexionaran acerca del peso del posicionamiento del traductor o traductora cuando se enfrenta a textos como los de la actividad preparada y si esta podía influir en la entrega final. En la segunda fase, una vez explicada la metodología de trabajo, cada estudiante debía proceder a la traducción del texto (véase Anexo 2). Para ello, trabajaron en clase con ordenadores y no se les hacía ningún tipo de recomendación de recursos lingüísticos, a excepción de la de textos paralelos que habían identificado en la fase anterior. Para la realización de la traducción, contaron con tres horas de clase. Finalmente, la tercera fase se completaba, una vez entregada la traducción, con otro cuestionario de preguntas abiertas para contrastar sus respuestas con las anteriores obtenidas previamente al proceso de trabajo (véase Anexo 3). De esta forma, nos interesaba el tratamiento de los términos, partes o expresiones del texto más difíciles de traducir después de haber realizado la traducción, los recursos que utilizaron, la influencia de la ideología a la hora de escoger la traducción de un término y, por último, si consideraban que era importante formar al estudiantado de traducción en este ámbito.

Para analizar las respuestas de los estudiantes, así como sus traducciones en base a los objetivos señalados con anterioridad, hemos seguido una metodología de análisis cualitativo aplicada por Roskoka y Rupniece (2016) en un estudio previo. Para ello hemos utilizado un marco de observación sobre unidades de contenido, agrupadas por categorías analíticas. Mostramos los resultados en el siguiente apartado en tablas específicas para cada categoría, de acuerdo con el modelo de literatura teórica de Geske y Grinfelds (2006). 
Martínez Pleguezuelos, A. J.; Alcalde Peñalver, E. y Santamaría Urbieta, A.

Retos ante la reescritura del cuerpo sexuado de la mujer y la prostitución femenina: estudio de caso

\section{RESULTADOS}

Tal y como mencionamos en el apartado anterior, analizaremos nuestros resultados en base a unidades de contenido, agrupadas por categorías analíticas, y mostraremos los resultados en tablas específicas para cada uno de los valores identificados. Marcamos, igualmente, con cada comentario, si se trataba de un alumno o una alumna.

Tabla 1. Opinión del estudiante sobre la influencia de la ideología (pre-test).

\begin{tabular}{|l|l|}
\hline Categoría de análisis & Unidades de contenido \\
\hline $\begin{array}{l}\text { Opinión del estudiante sobre la } \\
\text { influencia de la ideología del } \\
\text { traductor (test previo a la } \\
\text { traducción) }\end{array}$ & $\begin{array}{l}\text { Although a translation shouldn't be influenced by the } \\
\text { translator's opinion, it can happen that maybe words such } \\
\text { as "pimp", if the translator has a negative view, they could } \\
\text { use the word "putero" instead of "proxeneta". 2 answers } \\
\text { (2 alumnas) }\end{array}$ \\
$\begin{array}{l}\text { Depending on the translator's point of view, some terms } \\
\text { could be used with positive or negative connotations. } 4 \\
\text { answers (3 alumnas y 1 alumno) }\end{array}$ \\
$\begin{array}{l}\text { If the text is controversial, the translator tends to use the } \\
\text { terminology that adapts to his/her thoughts, although the } \\
\text { translator must be neutral. 6 answers (3 alumnas y } 3 \\
\text { alumnos) }\end{array}$ \\
\hline
\end{tabular}

Fuente: elaboración propia.

Estas respuestas se muestran en consonancia con los objetivos número dos y tres de nuestra investigación, ya que los estudiantes reflexionaron sobre el papel del traductor a la hora de escoger un término u otro en el texto en español y la influencia que la ideología sobre este tema tenía en el proceso.

Tabla 2. Opinión del estudiante sobre la influencia de la ideología (post-test).

\begin{tabular}{|l|l|}
\hline Categoría de análisis & Unidades de contenido \\
\hline $\begin{array}{l}\text { Opinión del estudiante sobre la } \\
\text { influencia de la ideología del } \\
\text { traductor (test posterior a la } \\
\text { traducción) }\end{array}$ & $\begin{array}{l}\text { The meaning of some words could have been less strong } \\
\text { but we decided to translate it as "dar la espalda", with a } \\
\text { stronger meaning, because of the meaning of the text. (1 } \\
\text { alumno) }\end{array}$ \\
$\begin{array}{l}\text { We don't think ideology played a role, since our objective } \\
\text { was always to stay as neutral as possible. 5 answers (3 } \\
\text { alumnos y 2 alumnas) }\end{array}$ \\
$\begin{array}{l}\text { We tried to avoid being biased, but we believe it was } \\
\text { inevitable to leave our print as translators. Sometimes we } \\
\text { had to resist the temptation of writing words with } \\
\text { connotations, but in the end we focused on translating the } \\
\text { author's ideas without including ours. (1 alumna) }\end{array}$ \\
\hline
\end{tabular}


Martínez Pleguezuelos, A. J.; Alcalde Peñalver, E. y Santamaría Urbieta, A.

Retos ante la reescritura del cuerpo sexuado de la mujer y la prostitución femenina: estudio de caso

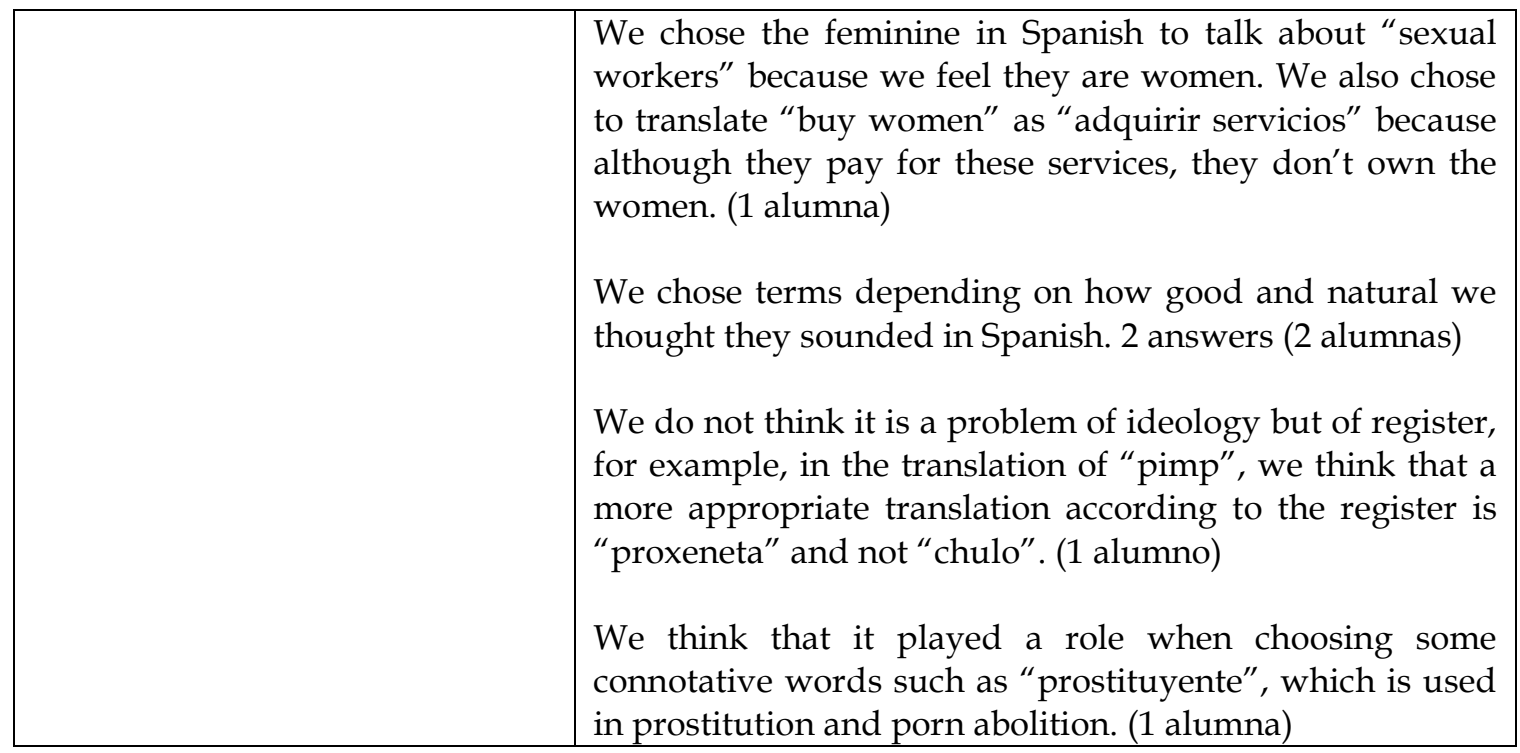

Fuente: elaboración propia.

En este caso, tras la realización de la traducción, los resultados muestran que los estudiantes fueron conscientes de que su opinión sobre el tema podía influir en sus decisiones de traducción. Esto se muestra en consonancia con los objetivos número dos y tres, puesto que los estudiantes reflexionaron a la hora de elegir los términos sobre la importancia que su opción léxica podía tener al transmitir el significado en español.

Tabla 3. Importancia de la formación del traductor en este ámbito.

\begin{tabular}{|l|l|}
\hline Categoría de análisis & Unidades de contenido \\
\hline $\begin{array}{l}\text { Importancia de la formación del } \\
\text { Translating this text helped us reflect on the deeper } \\
\text { meaning of some texts. (1 alumna) }\end{array}$ & $\begin{array}{l}\text { It is really important to know the meaning of specific } \\
\text { terms. } 3 \text { answers (2 alumnos y } 1 \text { alumna) }\end{array}$ \\
$\begin{array}{l}\text { We don't think specific training for this type of texts is } \\
\text { needed. } 2 \text { answers (1 alumna } 1 \text { alumno) }\end{array}$ \\
$\begin{array}{l}\text { It is important to know how to translate this kind of } \\
\text { controversial texts and try to stick to the original meaning } \\
\text { of the text. (1 alumna) } \\
\text { We believe it is very important that translators have } \\
\text { specific training in this field due to the importance of the } \\
\text { topic and the need to spread it in everyday language. (1 } \\
\text { alumna) } \\
\text { We think it is useful because this is a hot topic now. With } \\
\text { feminism growing into people's mind more issues like this } \\
\text { will need to be translated. (1 alumna) }\end{array}$ \\
\hline
\end{tabular}


Martínez Pleguezuelos, A. J.; Alcalde Peñalver, E. y Santamaría Urbieta, A.

Retos ante la reescritura del cuerpo sexuado de la mujer y la prostitución femenina: estudio de caso

\begin{tabular}{|l|l|}
\hline & $\begin{array}{l}\text { It is useful because it will help the translator to be more } \\
\text { specific and adapt to the clients' needs. } 3 \text { answers (3 } \\
\text { alumnas) }\end{array}$ \\
\hline
\end{tabular}

Fuente: elaboración propia.

Los estudiantes del Grado en Lenguas Modernas y Traducción son, como muestran las unidades de contenido plasmadas en la tabla 3, conscientes de la importancia de una correcta preparación en traducción en el ámbito que nos ocupa. Asimismo, algunas de las respuestas dan muestra de la necesidad e importancia de formar al traductor en temas de actualidad, así como de especialidad, con los que se van a encontrar en el futuro mundo profesional para el que se están formando.

En definitiva, podemos afirmar que los tres objetivos que se planteaban al comienzo del estudio de caso que nos ocupa ([1] conocer, [2] analizar y [3] generar concienciación) han sido alcanzados puesto que, en primer lugar, hemos planteado un encargo de traducción a estudiantes que se enfrentaban por primera vez a una tarea de este tipo. Hemos conocido, por un lado, desde qué punto de vista afrontaban la traducción de un texto en el que la perspectiva de género era esencial. Por otro lado, hemos podido analizar la posición temática que han tomado $\mathrm{y}$, finalmente, hemos generado concienciación acerca de la importancia de recibir formación específica y del papel que debe adoptar un traductor frente a un encargo de estas características.

En cuanto a los problemas de traducción del texto, los estudiantes optaron por diferentes soluciones para traducir algunas de las principales dificultades detectadas en función de la problemática planteada en nuestro estudio, cuyos ejemplos más representativos pasamos a mostrar. En el primer caso, para la traducción de "pimps and buyers of sex", las respuestas fueron todas iguales para "pimps" (proxenetas), pero difieren en el segundo término, tal y como se aprecia en el siguiente gráfico. Los saltos en la representación del cliente de la prostitución no serán cuestión baladí y repercutirá en la imagen que se muestra al lector meta en español. El énfasis puesto es la selección léxica, así como las diferencias apreciables entre "clientes" o "consumidores" y la aparición o ausencia de referencias directas a "sexo" o "prostitución" condicionarán la recepción del texto e influirán en el discurso en torno a la figura y participación del hombre en el mercado sexual, cuyo presencia y visibilidad demandan recientes enfoques feministas. Para oportuno señalar, además, que los alumnos varones tradujeron dicho término siempre como "clientes", mientras que el grupo de alumnas optaron por opciones más variadas. 
Martínez Pleguezuelos, A. J.; Alcalde Peñalver, E. y Santamaría Urbieta, A. Retos ante la reescritura del cuerpo sexuado de la mujer y la prostitución femenina: estudio de caso
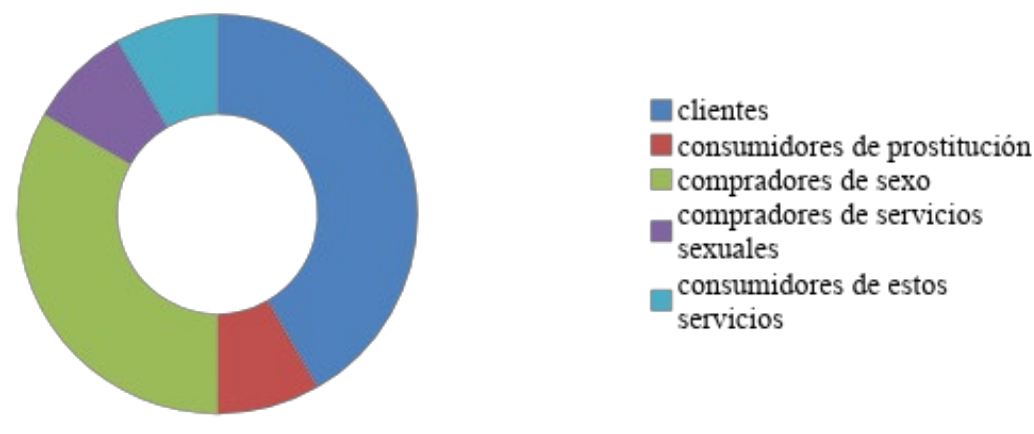

Gráfico 1: Traducción de "buyers of sex".

Fuente: elaboración propia.

En relación con el uso del masculino o el femenino para la traducción del término "sex workers" (gráfico 2) la mayoría de los estudiantes optaron por su uso en masculino. En cierta medida, y según el punto de vista que mantiene el propio texto original, resulta llamativo que la mayoría de los alumnos hayan optado por la versión en masculino, con la que la exposición de la mujer a la violencia estructural de la prostitución resulta parcialmente oculta tras un masculino genérico que no refleja la realidad del mercado sexual que describen los estudios sobre los que ya hemos hablado (Schulze, 2014). En este sentido, cabe señalar que ningún alumno varón empleó la forma en femenino en su traducción.

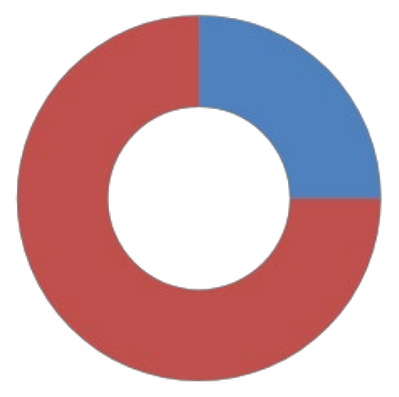

las trabajadoras sexuales los trabajadores sexuales

Gráfico 2: Traducción de "sex workers".

Fuente: elaboración propia.

En cuanto a la traducción del término "sex trade", los alumnos mostraron preferencia por el término "comercio sexual", mientras que en un caso optaron por "trata sexual". Las discrepancias semánticas que se aprecian entre las distintas opciones no escapan a nuestro análisis, y resulta necesario tener presente las asimetrías que se presentan al lector meta en uno y otro caso. La reminiscencia del término "trata" a la explotación, violencia y esclavitud de seres humanos, todo ello recogido en el Diccionario de la Real Academia Española, reafirma la crueldad y dureza de este tipo de mercantilización del cuerpo de la mujer en la sociedad actual. En este caso, salvo en la opción de "comercialización del sexo", únicamente empleado por alumnas, encontramos que tanto chicos como chicas han empleado el resto de soluciones en su traducción. 
Martínez Pleguezuelos, A. J.; Alcalde Peñalver, E. y Santamaría Urbieta, A.

Retos ante la reescritura del cuerpo sexuado de la mujer y la prostitución femenina: estudio de caso

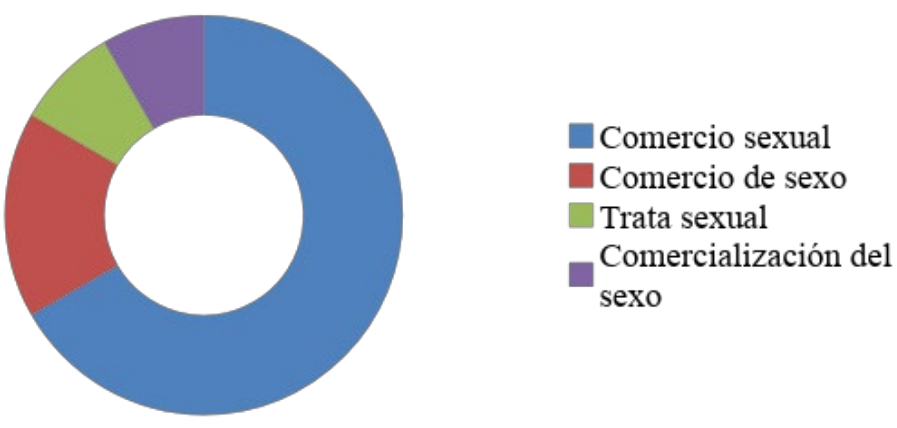

Gráfico 3: Traducción de "sex trade".

Fuente: elaboración propia.

En esta misma línea, para "sex trafficking" solo se han encontrado dos opciones, y la solución minoritaria, en sintonía con la traducción de "sex trade", sigue siendo la de "trata sexual". Este tratamiento terminológico en el discurso traducido viene a confirmar, como exponíamos en el marco teórico de nuestro estudio, una reducción en el tono del texto, donde la severidad del tema entra en conflicto con el panorama que la mayoría de los estudiantes desarrollan en su propuesta. Quizás, tal y como ya han tratado diversos autores (cf. Santaemilia, 2008; Ávila Cabrera, 2014), la aproximación a un tema tabú, influido en gran medida por la visión patriarcal predominante de la sociedad contemporánea, cercena las posibilidades discursivas de los estudiantes de Traducción, que aún no han interiorizado la necesidad de mantener el mismo mensaje. Señalamos que únicamente un alumno varón en el grupo de estudiantes ha utilizado el término "trata sexual", junto a otras tres alumnas.

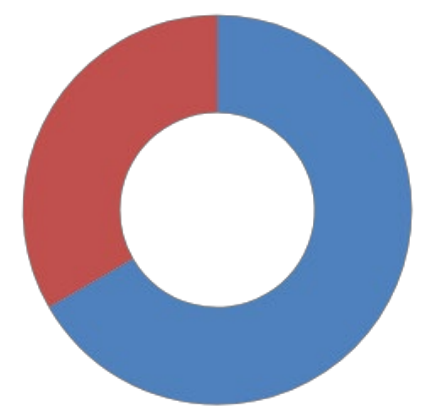

tráfico sexual trata sexual

Gráfico 4: Traducción de "sex trafficking".

Fuente: elaboración propia.

Por último, seleccionamos la oración "Those who derive sexual pleasure from reducing the humanity of women they buy" para analizar si de la interpretación de su contenido surgían distintas opciones de traducción. Por un lado, la más utilizada (seis casos) fue "aquellos que separan el placer sexual de reducir la humanidad de las mujeres que compran", es decir, mediante el uso de "reducir la humanidad" y "mujeres que compran". No obstante, encontramos un caso en el que omitieron "las mujeres que compran" y decidieron optar por "trabajadora sexual", tal y como se muestra a continuación: "los que obtienen placer sexual reduciendo la humanidad de la trabajadora sexual". No obstante, en los cuatro casos restantes observamos las siguientes traducciones del verbo "reducir": 
Martínez Pleguezuelos, A. J.; Alcalde Peñalver, E. y Santamaría Urbieta, A.

Retos ante la reescritura del cuerpo sexuado de la mujer y la prostitución femenina: estudio de caso

Traducción 1: "los que obtienen placer sexual al rebajar la humanidad de las mujeres que compran".

Traducción 2: "los que obtienen placer sexual negando la integridad de la mujer que compran".

Traducción 3: "aquellos que obtienen placer sexual rebajando la humanidad de la mujer que compran".

Traducción 4: "aquellos que obtienen placer sexual al humillar a las mujeres que compran".

Estos resultados revelan que es importante, tal y como señalaba Kim (2009), que la teoría de la traducción se centre en las personas, puesto que la investigación en estos ámbitos puede contribuir de manera específica a que la forma de redactar textos con perspectiva de género no se vea sesgada por la ideología predominante en la sociedad. Tal y como demuestran los trabajos sobre este aspecto (cf. Calzada, 2003), resulta prácticamente imposible que la ideología no se pronuncie a través del texto meta, es decir, que la opinión del traductor o traductora se vea reflejada a través del uso de determinado léxico, artículo o verbo. Incluso, tal y como afirma Pym (2010), las posiciones más neutrales deben ser creadas desde una selección lingüística concreta. Este autor, además, subraya el grado de responsabilidad que se debe adoptar en la traducción, lo cual debe evaluarse en todo momento en función de los textos en los que se trabaja. A esto añade que "translators are expected to be intellectuals who have ideas about their collective identity and aims" (Pym, 2010, p. 179). Por lo tanto, resulta de vital importancia informar y formar a los profesionales del futuro sobre la traducción de textos complejos de profundo calado social que pueden llegar a tener repercusiones en la vida de distintos colectivos. En raras ocasiones se enfrentarán a traducciones de carácter objetivo, ya que deberán hacer frente a encargos que les hagan reflexionar sobre su propia opinión frente al tema que se aborde. Hacerse partícipes del discurso a través del uso de determinados recursos del lenguaje o invisibilizarse ante los ojos del lector del texto meta será una decisión que ellos mismos deban tomar. Aunque adoptar una $u$ otra postura sea el paso final al que se deban enfrentar, se constituye como el punto de partida para el docente, quien debe proporcionar todas las herramientas y posibilidades al alcance de estos futuros profesionales.

\section{DISCUSIÓN}

Tras el estudio de caso presentado y el análisis de los resultados obtenidos, corroboramos las implicaciones éticas del ejercicio de la traducción en la definición discursiva tanto de la mujer como del hombre en sentidos opuestos. Por un lado, comprobamos que, al hablar de prostitución, continúa pasándose por alto el papel del hombre como cliente y solicitante de un mercado que, pese a los avances legislativos y sociales, va en aumento. El vínculo entre comercio sexual y el cuerpo de la mujer sigue presente en las reescrituras con casos, por ejemplo, como el de la elección por gran parte del alumnado de la expresión "los trabajadores sexuales" en lugar de "las trabajadoras sexuales". La neutralización del femenino en esta construcción invisibiliza la violencia que se ejerce fundamentalmente de manera 
Martínez Pleguezuelos, A. J.; Alcalde Peñalver, E. y Santamaría Urbieta, A.

Retos ante la reescritura del cuerpo sexuado de la mujer y la prostitución femenina: estudio de caso

unidireccional hacia la mujer y no señala, para el lector meta, quién es la víctima en la inmensa mayoría de casos de prostitución.

Por otra parte, y en línea con este argumento, el poder de la ideología ejerce presión sobre el traductor o traductora cuando debe recrear la identidad de la mujer en el discurso de la prostitución y contribuirá a que, de manera consciente o inconsciente, ofrezca una imagen determinada en su traducción. Por ello, según los resultados obtenidos, y a la vista del enorme potencial con el que cuentan estos profesionales, consideramos necesario seguir formando al alumnado en traducción para que sea capaz de detectar las herramientas discursivas que contribuyen a afianzar los desequilibrios entre hombres y mujeres y los roles que quedan asignados en la sociedad.

\section{REFERENCIAS}

Ávila Cabrera, J. J. (2014). The Subtitling of Offensive and Taboo Language: A Descriptive Study. Tesis doctoral: Universidad Nacional de Educación a Distancia.

Basnett, S. \& Lafevere, A. (Eds.) (1990). Translation, History \& Culture. London: Printer Publishers.

Burgos Díaz, E. (2006). Cuerpos que hablan. Contrastes. Revista internacional de filosofía, 11(1), 93-109.

Butler, J. (1999/2007). El género en disputa. El feminismo y la subversión de la identidad. Barcelona: Paidós.

Calzada Pérez, M. (Ed.) (2003). Apropos of Ideology. Translation Studies on IdeologyIdeologies in Translation Studies. Manchester: St. Jerome.

Cronin, M. (2006). Translation and Identity. London/New York: Routledge.

De Mauro Rucovsky, M. A. (2015). Cuerpos en escena. Materialidad y cuerpo sexuado en Judith Butler y Paul B. Preciado. Madrid: Egales.

De Miguel Álvarez, A. (2012). La prostitución de mujeres, una escuela de desigualdad humana. Revista europea de derechos fundamentales, 19(1), 49-74.

Ekberg, G. (2004). The Swedish Law that Prohibits the Purchase of Sexual Services. Best Practices for Prevention of Prostitution and Trafficking in Human Beings. Violence Against Women, 10(10), 1187-1218.

Farley, M. (2004). Bad for the Body, Bad for the Heart: Prostitution Harms Women Even if Legalized or Decriminalized. Violence Against Women, 10(10), 1087-1125. 
Martínez Pleguezuelos, A. J.; Alcalde Peñalver, E. y Santamaría Urbieta, A.

Retos ante la reescritura del cuerpo sexuado de la mujer y la prostitución femenina: estudio de caso

Gentzler, E. (2017). Translation and Rewriting in the Age of Post-Translation Studies. London/Yew York: Routledge.

Gentzler, E. (2012). Translation without Borders» en Translation: A Transdisciplinary Journal. Recuperado de http: //translation.fusp.it/articles/translation-without$\underline{\text { borders }}$

Gentzler, E. (2002). Translation, Poststructuralism, and Power, en TYMOCZKO, María y Edwin GENTZLER (Eds.) Translation and Power. Amherst/Boston: University of Massachusetts Press (pp. 195-218). Recuperado de http://lstlss.ucl.ac.uk/course-materials/ELCS6078_73800.pdf

Geske, A. \& Grinfelds, A. (2006). Education research. Riga: LU.

Gimeno, B. (2014). La prostitución tiene que ver con la igualdad, no con el sexo, en Eldiario.es. Recuperado de https://www.eldiario.es/zonacritica/prostitucion-verigualdad-sexo_6_235936431.html

House, J.; Martín Ruano, R. \& Baumgarten, N. (Eds.) (2005). Translation and the Construction of Identity. Seoul: IATIS.

Kim, Sh. (2009). Towards a People-Centered Theory of Translation». Perspectives: Studies in Translatology, 17(4), 257-272.

Mackinnon, C. A. (2011). Trafficking, Prostitution and Inequality. Harvard Civil Rights-Civil Liberties Law Review, 46(8), 271-309.

Mackinnon, C. A. (2005). Pornography as Trafficking. Michigan Journal of International Law, 26(4), 993-1012. Recuperado de https:// repository.law.umich.edu/cgi/viewcontent.cgi?article $=1241 \&$ context $=$ mjil

Pym, A. (2010). Translation and Text Transfer: An Essay on the Principles of Intercultural Communication. Tarragona: Grupo de Estudios Interculturales.

Raymond, J. G. (1999). Legitimating Prostitution as Sex Work: UN Labour Organization (ILO) Calls for Recognition of the Sex Industry. United States. Recuperado de http:// www.catwinternational.org/Home/Article/61-legitimating-prostitutionas-sex-work-un-labour-organization-ilo-calls-for-recognition-of-the-sex-industry Consultado el 31/03/19

Rodríguez Arcos, I. (2018). Women's Magazines and Symbolic Violence: The Translation of the Body in the Era of Mass Communications, en HIBBSLISSORGUES, Solange; ŞERBAN, Adriana y Nathalie VINCENT-ARNAUD (Eds.) The body and translation, the body in translation (translation and conference interpreting). 
Martínez Pleguezuelos, A. J.; Alcalde Peñalver, E. y Santamaría Urbieta, A.

Retos ante la reescritura del cuerpo sexuado de la mujer y la prostitución femenina: estudio de caso

Roskosa, A. \& Rupniece, D. (2016). Advantages and drawbacks of using group work in translator training. Procedia. Social and Behavioral Sciences, 231, 244-250. Recuperado de https:/ / www.sciencedirect.com/science/article/pii/S1877042816312095

Santaemilia, J. (2008). The translation of sex-related language: the danger(s) of selfcensorship(s). TTR: Traduction, Terminologie, Rédaction, 21(2), 221-252.

Schulze, E. (2014). Sexual Exploitation and prostitution and its impact on gender equality. Bruselas: Directorate General for Internal Policies. Policy Department C: Citizens' Rights and Constitutional Affairs. Recuperado de http:// www.europarl.europa.eu/RegData/etudes/etudes/join/2014/493040/IP OL-FEMM_ET(2014)493040_EN.pdf

Tymoczko, M. (2007). Enlarging Translation, Empowering Translators. Amherst/Boston: University of Massachusetts Press.

Vidal Claramonte, M. C. A. (2018). The body as a Semiotic System of Representation en FEDERICI, Eleonora y Marilena PARLATI (Eds.), The Body Metaphor, 5, 17-26.

Vidal Claramonte, M. C. A. (2007). Traducir entre culturas: poderes, diferencias, identidades. Frankfurt am Main: Peter Lang.

Villa Camarma, E. (2010). Estudio antropológico en torno a la prostitución. Cuicuilco. Revista de la Escuela Nacional de Antropología e Historia, 17(49). Recuperado de http:/ / www.scielo.org.mx/scielo.php?script=sci_arttext\&pid=S0185$\underline{16592010000200009}$

\section{AUTORES:}

\section{Antonio Martínez Pleguezuelos}

Licenciado en Traducción e Interpretación por la Universidad de Granada, máster en Traducción y Mediación Intercultural y doctor en Traducción e Interpretación por la Universidad de Salamanca. En la actualidad es Profesor Asociado tanto en la Universidad Complutense de Madrid como en la Universidad Autónoma de Madrid. Sus líneas de investigación incluyen traducción, ideología e identidad, traducción y estudios de género, sexualidad y Teoría Queer y traducción audiovisual, ámbitos sobre los que ha publicado distintos capítulos y artículos en editoriales y revistas nacionales e internacionales (Meta, MonTI, Trans, Atlantis, Quaderns de Filología, etc.). Es autor del libro Traducción e identidad sexual: reescrituras audiovisuales desde la Teoría Queer (Granada: Comares, en prensa). Combina la docencia y la investigación con la traducción profesional como traductor autónomo.

antmar17@ucm.es

Orcid ID: $\underline{\text { https://orcid.org/0000-0002-1732-3178 }}$

Google Scholar: https:// scholar.google.es/citations?user=NjFom9QAAAAJ\&hl=es ResearchGate:

https://www.researchgate.net/profile/Antonio_Martinez_Pleguezuelos 
Martínez Pleguezuelos, A. J.; Alcalde Peñalver, E. y Santamaría Urbieta, A.

Retos ante la reescritura del cuerpo sexuado de la mujer y la prostitución femenina: estudio de caso

\section{Elena Alcalde Peñalver}

Elena Alcalde Peñalver obtuvo el doctorado por la Universidad de Granada en 2014, tras haber disfrutado de una beca FPU en el Departamento de Traducción e Interpretación de esta universidad. Es también Licenciada en Traducción e Interpretación por la Universidad de Granada en la combinación lingüística inglésfrancés-español. Desde septiembre de 2017 es profesora Ayudante Doctora en la Universidad de Alcalá. Además, desde 2010 ejerce como traductora e intérprete independiente.

e.alcalde@uah.es

Orcid ID: http:// orcid.org/0000-0002-1606-4792

Google Scholar: https:// scholar.google.es/ citations?user=Pp4ONR8AAAAJ\&hl=es

ResearchGate: https://www.researchgate.net/profile/Elena_Alcalde_Penalver

\section{Alexandra Santamaría Urbieta}

Doctora por la Universidad de Las Palmas de Gran Canaria y trabaja actualmente como docente e investigadora en la Universidad de Alcalá. Es licenciada en Traducción e Interpretación por la Universidad del País Vasco.

alexandra.santamaria@uah.es

Orcid ID: https:/ / orcid.org/0000-0003-0935-0616

Google Scholar: https://scholar.google.es/citations?user=Em8ZzYUAAAAJ\&hl=es

ResearchGate: A-9675-2018 
Martínez Pleguezuelos, A. J.; Alcalde Peñalver, E. y Santamaría Urbieta, A.

Retos ante la reescritura del cuerpo sexuado de la mujer y la prostitución femenina: estudio de caso

\section{ANEXO 1: Cuestionario previo a la realización de la traducción}

Work in pairs. You are going to translate this text into Spanish. Before starting to translate, please answer the following questions.

1. Read the text carefully. In your view and before consulting any kind of linguistic resources, what parts/terms/expressions of the text do you think that will be difficult to translate? Justify your answer.

2. Look for parallel texts in Spanish on the same topic. What terms can you already translate using those parallel texts without any other linguistic resources? Write the term in English, the translation you found in Spanish and the source of the parallel text.

\begin{tabular}{|l|l|l|}
\hline $\begin{array}{l}\text { Term from the text in } \\
\text { English }\end{array}$ & $\begin{array}{l}\text { Term from the parallel } \\
\text { text in Spanish }\end{array}$ & $\begin{array}{l}\text { Source of the parallel } \\
\text { text }\end{array}$ \\
\hline & & \\
\hline & & \\
\hline
\end{tabular}

3. Are there any terms that could be translated in different ways (synonyms or different expressions) in Spanish? If you had to choose one before starting to translate, which one would it be? Write them in the different columns.

\begin{tabular}{|l|l|l|}
\hline $\begin{array}{l}\text { Term from the text in } \\
\text { English }\end{array}$ & $\begin{array}{l}\text { Different options in } \\
\text { Spanish }\end{array}$ & Chosen term \\
\hline & & \\
\hline & & \\
\hline
\end{tabular}

4. Do you think that the translator's ideology on a controversial topic such as the one covered in the text that you are going to translate can have an influence on the result of the final translation? Why? 
Martínez Pleguezuelos, A. J.; Alcalde Peñalver, E. y Santamaría Urbieta, A.

Retos ante la reescritura del cuerpo sexuado de la mujer y la prostitución femenina: estudio de caso

\section{ANEXO 2: Texto para traducir}

Amnesty International says prostitution is a human right - but it's wrong If the organization endorses prostitution as a human right, it won't be supporting the women who might have no choice, but rather the pimps and buyers of sex who have all the choice in the world

Jessica Neuwirth

Has Amnesty International been hijacked by proponents of the global sex trade? When the human rights nonprofit convenes its International Council Meeting next week in Dublin, delegates from around the world will be asked to vote on a proposal to recognize prostitution as a human right.

Amnesty is arguing that prostitution is a matter of free choice, a stance heavily promoted by the multibillion-dollar commercial sex industry. The group is putting forth the view that sex work is compatible with the principle of gender equality and nondiscrimination, as if it were a job like any other.

"By definition," Amnesty's proposal states, "sex work means that sex workers who are engaging in commercial sex have consented to do so." This definition fails to take into account the dire economic need, the childhood sexual abuse, the brutal coercion employed by pimps, and the vast power differences of sex and race that drive the commercial sex industry.

Amnesty contends that "such conditions do not inevitably render individuals incapable of exercising personal agency". This argument ignores the reality for the vast majority of individuals exploited by the commercial sex industry. When United Nations personnel trade food for sex, these transactions - called "survival sex" might technically be consensual, but can hardly be considered examples of free will. Almost all prostitution is some form of survival sex. There is no choice in the absence of the freedom to choose otherwise.

Amnesty's stance on prostitution shows it is missing a gender lens. This isn't the first time Amnesty has been slow to protect women's rights: the group failed even to recognize sex trafficking as a human rights violation until the late 1990s. But it now recognizes rape as a weapon of war and some other forms of violence against women - including trafficking - as violations of human rights.

It also previously shied away from recognizing female genital mutilation as a human rights violation, noting the practice was deeply rooted in tradition, but then took up the issue in 1995. The issue is harm, not choice.

Perhaps Amnesty should look to the 1949 UN Convention on trafficking, which characterizes prostitution and sex trafficking as "incompatible with the dignity and worth of the human person and endanger[ing] the welfare of the individual, the 
Martínez Pleguezuelos, A. J.; Alcalde Peñalver, E. y Santamaría Urbieta, A.

Retos ante la reescritura del cuerpo sexuado de la mujer y la prostitución femenina: estudio de caso

family and the community". If the organization endorses prostitution as a human right, it won't be supporting the women who might have no choice but to have sex for money, but rather the pimps and buyers of sex who have all the choice in the world.

Amnesty is urging its membership to separate prostitution and sex trafficking as entirely unrelated. Yet common sense and the economics of supply and demand dictate that demand for prostitution fuels sex trafficking to supply it: not all prostituted women are sex trafficking victims, but all sex trafficking victims are sold into prostitution. Amnesty is urging its membership to legalize the industry, making no distinction between the women being prostituted and those who pay for and profit from their exploitation.

Sweden has made a legal distinction between those driven into the sex industry by poverty and discrimination and those who buy sex as an exercise of power and privilege. Its model law criminalizes only the buying of sex and offers support services to those who are bought. This progressive feminist method aims to decriminalize prostituted women without legitimizing the men who buy them.

In the book Paid For, a compelling analysis of author Rachel Moran's experience in the sex trade, she describes three types of men who patronize prostitution: those who assume the women they buy have no human feelings; those who are conscious of a woman's humanity but choose to ignore it; and those who derive sexual pleasure from reducing the humanity of women they buy. Is Amnesty really going to defend the rights of such men to buy women?

It's a heartbreaking moment for those of us who love Amnesty International. Former US president Jimmy Carter, who made human rights a centerpiece of US foreign policy, has started an online petition urging the group not to endorse commercial sexual exploitation as a right. The concept of human rights itself - not to mention sex equality - is at stake.

746 words

Source:

https:/ / www.theguardian.com/sustainable-business/2015/jul/28/amnestyinternational-prostitution-sex-work-human-trafficking 
Martínez Pleguezuelos, A. J.; Alcalde Peñalver, E. y Santamaría Urbieta, A.

Retos ante la reescritura del cuerpo sexuado de la mujer y la prostitución femenina: estudio de caso

\section{ANEXO 3: Cuestionario posterior a la realización de la traducción}

1. After completing the translation, which were the most difficult terms/parts/expressions to translate? Justify your answer.

2. Which resources were the most useful to solve the translation problems that you found in the text?

3. Do you think that your ideology on the topic played a role in choosing one term in Spanish over another? Justify your answer.

4. Do you think that specific translation training for texts on topics such as the one covered in the article would be useful for translators? Why? 